\title{
Analysis of AODV Protocol in MANET
}

\author{
Purba Pal \\ B.Tech IV Year \\ Dept. of CSE \\ TIT Narsinghar
}

\author{
Priya Sarkar \\ B.Tech IV Year \\ Dept. of CSE \\ TIT Narsinghar
}

\author{
Sonali Deb \\ B.Tech IV Year \\ Dept. of CSE \\ TIT Narsinghar
}

\author{
Gourab Bhattacharya \\ Assistant Professor \\ Dept. of CSE \\ TIT Narsinghar
}

\begin{abstract}
The Ad-Hoc on demand distance vector (AODV) routing protocol to be used by mobile nodes in an ad hoc network. It offers fast adaptation to dynamic link conditions, low process and memory overhead, low network utilization and determines unicast routes destinations inside the ad hoc network. It uses destination sequence numbers to confirm loop freedom in any respect of times (even within the face of abnormal delivery of routing management messages), avoiding issues (such as "continuity to infinity") related to classical distance vector protocols. Ad-Hoc networking could be a thought in pc communications characterized by property through a set of wireless nodes and fast changing network topology. In a wireless scenario, nodes are free to move hence maintaining path (route) is a difficult task. Routing protocols have central role in a wireless scenario. We have analyzed AODV protocol by extensive simulations in ns-2 simulator with various performance matrices such as Throughput, Number of packets sent, Number of packets received, Packets delivery ratio, Control overhead, Packet dropping ratio, Delay, Normalized routing overhead under wireless scenarios varying speed. And with this we are also trying to detect the Black Hole Attack in AODV.
\end{abstract}

\section{General Terms}

Ad Hoc Networking.

\section{Keywords}

AODV, MANET, ns-2 simulator, Packet delivery fraction, Throughput, Number of packets sent, Number of packets received, Packet delivery ratio, Control over head, Packet dropping ratio, Delay, Normalized routing overhead.

\section{INTRODUCTION}

\subsection{MANET}

MANET stands for Mobile adhoc Network additionally known as wireless adhoc network or adhoc wireless network that typically contains a routable networking atmosphere on top of a Link Layer ad hoc network.. They carries with itset of mobile nodes connected wirelessly during a self designed, self healing network while not having a fixed infrastructure. Manet nodes are unengaged to move every which way because the topology changes oftentimes. Every node behave as a router as they forward traffic to alternative given node within the network. MANET might operate as standalone fashion or they can be the part of larger internet. They form extremely dynamic autonomous topology with the presence of 1or multiple completely different transceivers between nodes.The main challenge for the MANET is to equipped every devices to incessantly maintain the information needed to properly route traffic. MANETs consist of a peer-to-peer, self-forming, self-healing network MANET's circa 2000-2015 usually communicate at radio frequencies $(30 \mathrm{MHz}-$ $5 \mathrm{GHz}$ ). This can be used in road safety, starting from sensors for environment, home, health, disaster rescue operations, air/land/navy defense, weapons, robots, etc.

\subsection{AODV}

An Ad Hoc On-Demand Distance Vector (AODV) is a routing protocol made for wireless and mobile ad hoc networks. This protocol creates routes to destinations on demand and supports both unicast and multicast routing. The AODV protocol was jointly designed by Nokia Research Center, the University of California, Santa Barbara and the University of Cincinnati in 1991.The AODV protocol builds routes between nodes when requested by source nodes. That's why AODV is considered as an on-demand algorithm and does not create any extra traffic for communication along links. The routes are maintaned as long as they are needed by the sources. AODV makes use of sequence numbers to make sure route freshness. They're self-starting and loop-free besides scaling to various mobile nodes. In AODV, networks are silent till connections are established. Network nodes broadcast a request for connection when need connections. The remaining AODV nodes forward the message and record the node that requested an affiliation. Thus, they produce a series of temporary routes back to the requesting node. A node that receives such messages and holds a route to a desired node sends a backward acknowledgment message through temporary routes to the requesting node. The node that initiated the request uses the route containing the least number of hops through alternative nodes. The entries that are not utilized in routing tables are recycled after some period of time. If a link fails, the routing error is passed back to the transmittal node and also the method is continued.

\subsection{Working of AODV}

When a node wishes to send a packet to some destination, it checks its routing table to determine if it has a current route to the destination. If yes, forwards the packet to next hop node If No, it initiates a route discovery process [8].

\subsubsection{Route discovery}

It begins with broadcasting of RREQ to its neighbors specified for certain destination. Once an intermediate node receives a RREQ, It check its routing table for route to dest If found send RREP to source If not found it rebroadcast RREQ to its neighbor nodes by setting up a reverse route path to source node in its route table. It ignores RREQ if it is processed already [6]. Finally on reaching RREQ to destination node, It uncast RREP to source node by using reverse route to source node. The above procedure can be described visually as follows: 


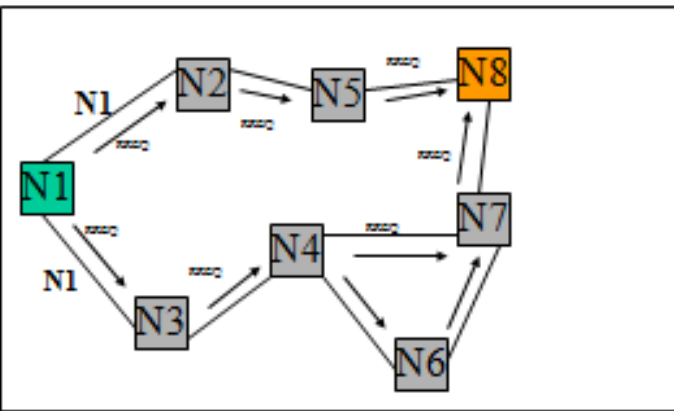

Fig 1: Route Discovery

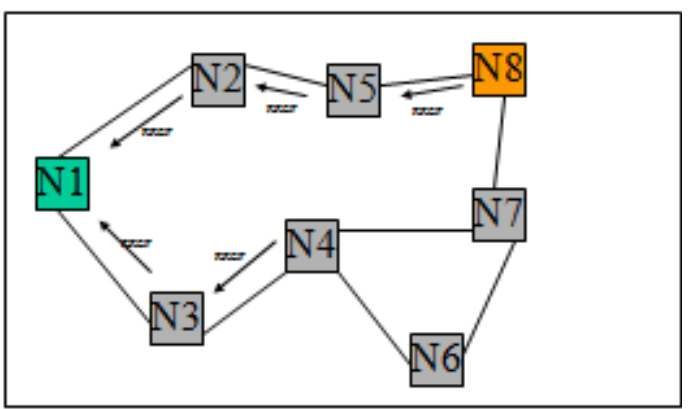

Fig 2: Route Reply

\subsubsection{Route Maintenance Stage}

A hello message is broadcasted by active nodes periodically. If no hello message from a neighbor The upstream node will notify the source with an RERR packet \& entire routes based on the node is invalidated. Source will initialize a new route discovery stage and flood the RREQ packet [8]. Above procedure can be realized in the following figure:

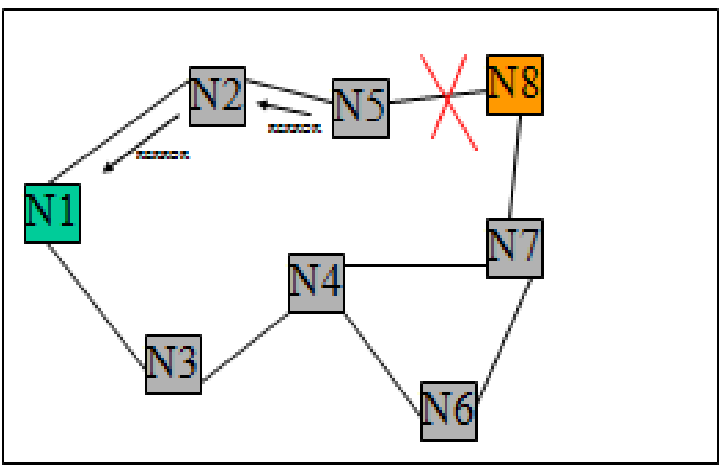

Fig 3: Propagation of RERR

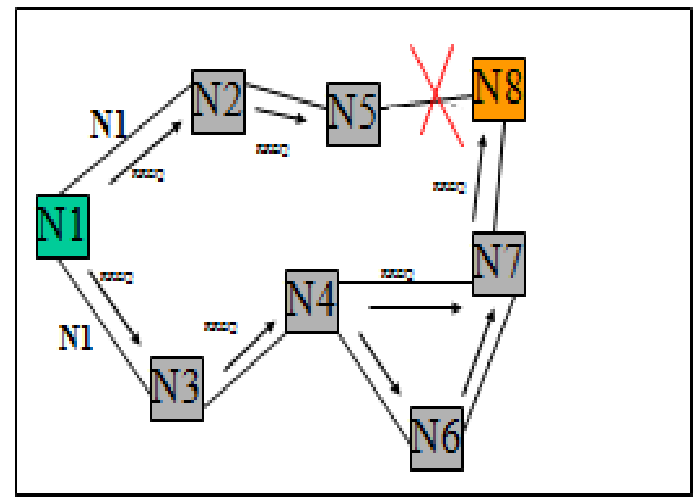

Fig 4: Route Rediscovery

\section{EVALUATION CRITERIA}

Performance of AODV protocols in MANET can be realized by quantitative study of values of different metrics used to measure performance of routing protocols which are as follows.

No of packet received : Total number of received packet.

No of packet sent: Total number of sending packet.

Packet delivery ratio(PDR): Packet delivery ratio is the ratio of total number of data packets received at destination to the total number of data packets sent from source.

PDR=Received packet/Packet sent $* 100$

Control overhead: Control overhead means the number of routing packets which are required in the network for data transmission.

\section{Control overhead=Routed Packet (rtr)}

Normalized routing overhead (NRO): Normalized routing overhead is the ratio of number of routing packets to the total number of data packets received.

$\mathrm{NRO}=\mathrm{rtr} /$ packet received $* 100$

Delay: Delay is the time taken by packet to reach to destination from source.

Delay $=$ delay/packet received

Throughput: Throughput means the average number of bits transmitted per unit time.

Throughput $=$ bytes $* 8 /$ (End time of packet transmissionStarting time of packet transmission)/1000)

Packet Dropping Ratio (PDR): Packet dropping ratio is the ratio of total number of data packets dropped to the total number of data packets sent from source.

$\mathrm{PDR}=($ packet sent-packet received $) /$ packet sent*100).

\section{SIMULATION MODEL}

For the simulation of the developed system, latest version 2.34 of NS-2 has been used in this paper. NS2 is an opensource simulation tool that runs on Linux.It's a discreet event tool targeted at networking analysis and provides substantial support for simulation of routing, multicast protocols and IP protocols over wired and wireless (local and satellite) networks.It is mainly used in research works. The first version was NS-1 and it was developed at Lawrence Berkeley National Laboratory (LBNL) in the 1995-97.Then came NS-2 as a revision of NS-1.Then came NS-3 and NS-4.

\section{SIMULATION PARAMETERS}

There are number of simulation parameters which can be varied, results in change in value of different performance metrics, which can be shown in below table.

Table 1. Simulation Parameter

\begin{tabular}{|c|c|c|}
\hline SI No & Parameter & Value \\
\hline $\mathbf{1}$ & Simulator & NS-2 (Version 2.34) \\
\hline $\mathbf{2}$ & Channel type & $\begin{array}{c}\text { Channel/Wireless } \\
\text { channel }\end{array}$ \\
\hline
\end{tabular}




\begin{tabular}{|c|c|c|}
\hline 3 & Radio Propagation Model & $\begin{array}{l}\text { Propagation/Two ray } \\
\text { ground wave }\end{array}$ \\
\hline 4 & Network interface type & Phy/WirelessPhy \\
\hline 5 & MAC Type & Mac /802.11 \\
\hline 6 & Interface queue Type & Queue/Drop Tail \\
\hline 7 & Link Layer Type & LL \\
\hline 8 & Antenna & Antenna/Omni Antenna \\
\hline 9 & Area $\left(M^{*} M\right)$ & $500 * 500$ \\
\hline 10 & Simulation Time & 30 \\
\hline 11 & No of Nodes & $10-100$ \\
\hline 12 & Routing Protocol & AODV \\
\hline
\end{tabular}

\section{SIMULATION SCENARIO}

There can be the possibility of following two scenarios shown in fig $5 \& 6$ below one is, static where nodes are constant \& another is dynamic where nodes are moving continuously which is consider in this paper.

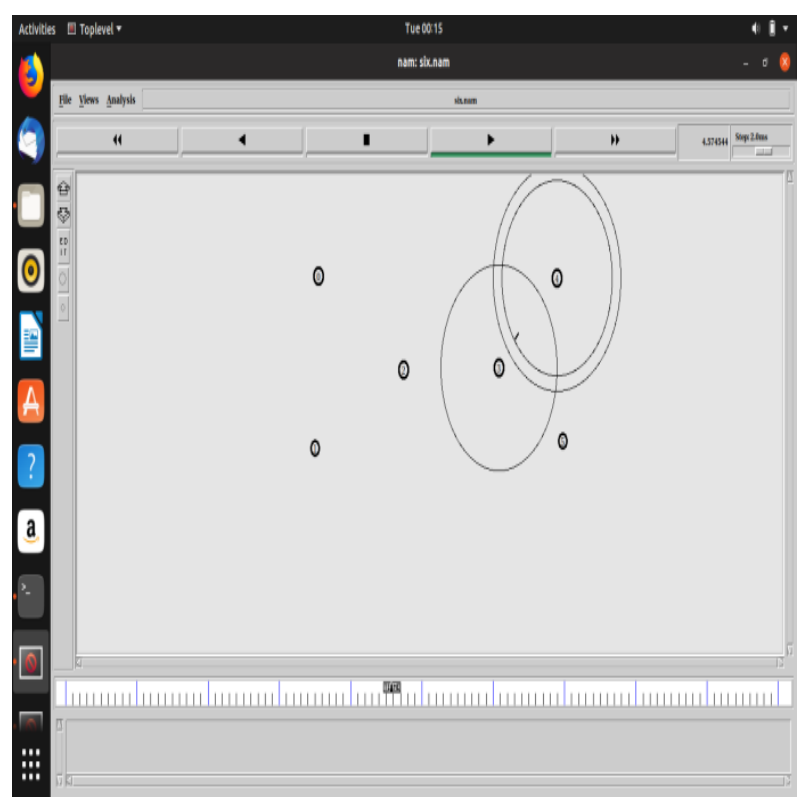

Fig 5: Static Simulation Scenario

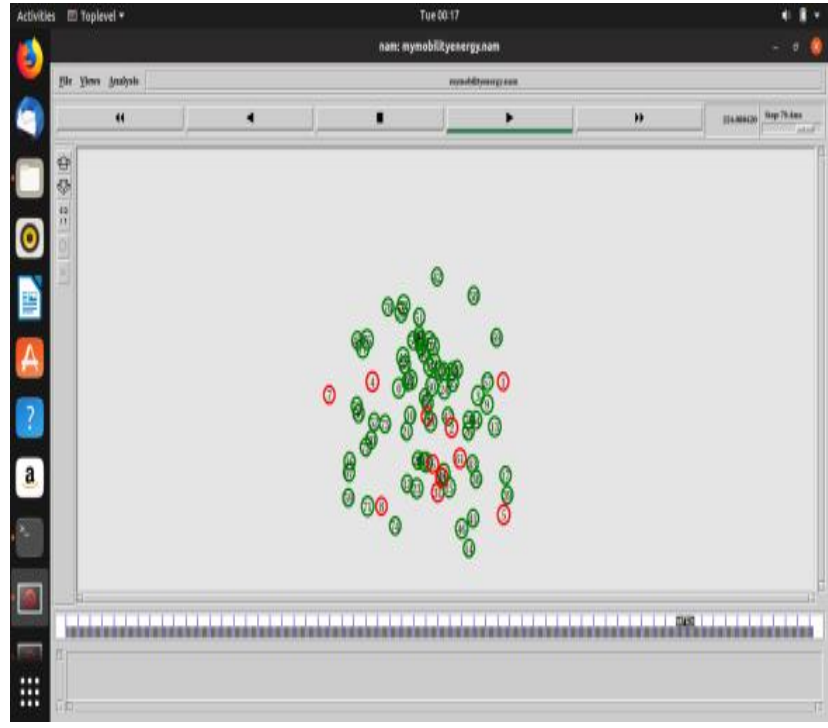

Fig 6: Dynamic Simulation Scenario

\section{RESULT AND ANALYSIS}

Using Random Waypoint Mobility Model analysing by increasing the number of nodes upto 100 to check the performances of the AODV protocol in terms of some metices given below varying speed.

\subsection{For Throughput}

Table 2. Simulation Result for Throughput

Fio of nodes speedl speed3 speeds speed7 speedlo

\begin{tabular}{|c|c|c|c|c|c|}
\hline 10 & 65.15 & 04.2 & 79.85 & 70.06 & 69.2 \\
\hline 20 & 5139 & 80.99 & 6677 & 70.2 & 67.2 \\
\hline 30 & 6.81 & 76.07 & 80.42 & 78.91 & 04.42 \\
\hline 40 & 74.59 & 75.22 & 79.66 & 76.47 & 68.8 \\
\hline 50 & 78.82 & 65.89 & 84:12 & 0.52 & 73.72 \\
\hline 60 & 45.97 & 77.2 & 78.87 & 67,03 & 77.99 \\
\hline 70 & 71.48 & 719 & 78.44 & 78.78 & 59.99 \\
\hline 90 & 67.81 & 75.93 & 67.31 & 74,54 & 79.66 \\
\hline 90 & $\pi, 4 \overline{1}$ & 76.78 & 3601 & 78.72 & 71.61 \\
\hline 100 & 77.8 & 40 & 74.45 & 撸自7 & 71.77 \\
\hline
\end{tabular}

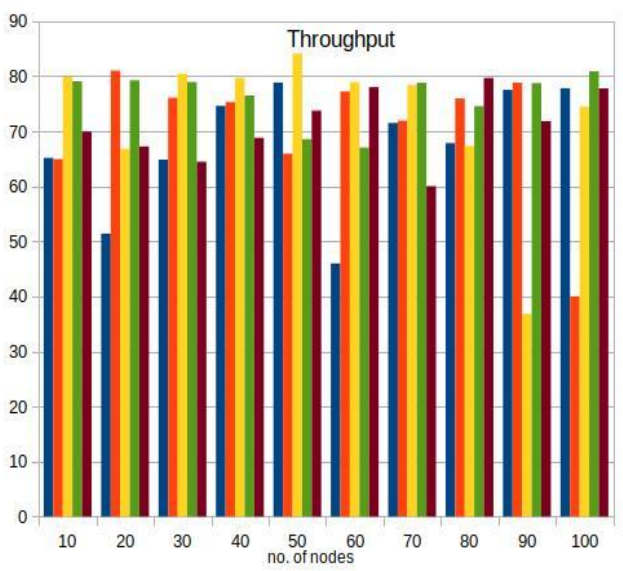

- speed1

Fig 7: Simulation graph for Throughput 


\subsection{For No of Packet Received}

Table 3. Simulation Result for No of packet received

no. of nodes speed1 speed3 speed5 speed7 speed10

\begin{tabular}{rrrrrr}
\hline 10 & 3552 & 4413 & 6469 & 6043 & 5787 \\
\hline 20 & 3759 & 4842 & 5057 & 5732 & 5857 \\
\hline 30 & 3363 & 4848 & 4653 & 6959 & 4559 \\
\hline 40 & 3771 & 4100 & 6025 & 4385 & 4338 \\
\hline 50 & 5472 & 4029 & 7852 & 4844 & 4103 \\
\hline 60 & 4293 & 3926 & 6066 & 3893 & 5677 \\
\hline 70 & 3521 & 4664 & 6034 & 4909 & 5602 \\
\hline 80 & 4283 & 5047 & 4994 & 3377 & 6643 \\
\hline 90 & 4884 & 5553 & 3436 & 5638 & 4728 \\
\hline 100 & 5426 & 3735 & 4974 & 5400 & 6531 \\
\hline
\end{tabular}

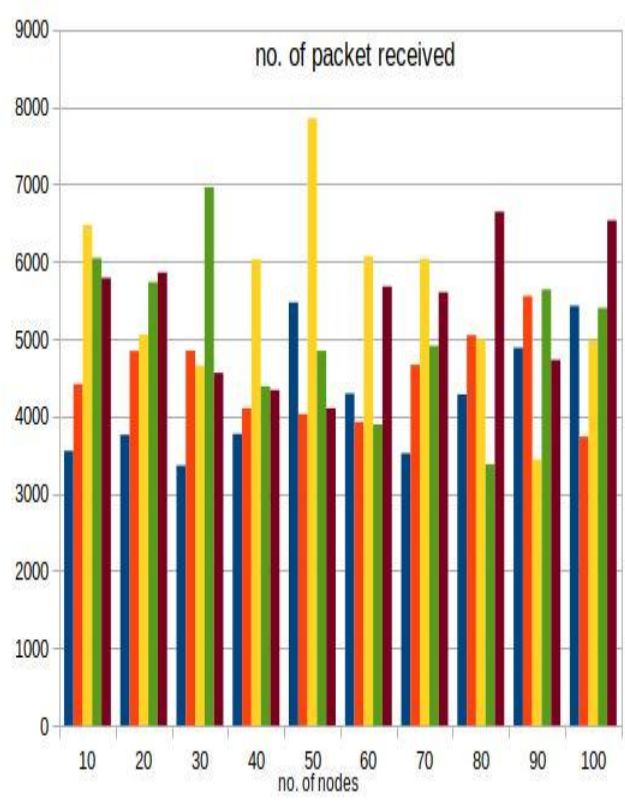

Ispeed1

II speed3

॥ speed5

Ispeed7

Ispeed10

Fig 8: Simulation graph for No of packet received

\subsection{For No of Packet Sent}

Table 4. Simulation Result for No of packet sent

\begin{tabular}{rrrrrr}
\hline no. of nodes speed1 & \multicolumn{2}{c}{ speed3 } & \multicolumn{2}{c}{ speed5 } & \multicolumn{2}{c}{ speed7 } & \multicolumn{2}{c}{ speed10 } \\
\hline 10 & 3734 & 4788 & 6881 & 6447 & 6466 \\
\hline 20 & 5019 & 4886 & 5832 & 6054 & 6775 \\
\hline 30 & 3857 & 5159 & 4711 & 7520 & 5219 \\
\hline 40 & 3889 & 4303 & 6347 & 4580 & 4609 \\
\hline 50 & 5740 & 4500 & 8262 & 5350 & 4335 \\
\hline 60 & 6048 & 3996 & 6539 & 4193 & 6033 \\
\hline 70 & 3755 & 5124 & 6526 & 5060 & 7000 \\
\hline 80 & 4647 & 5378 & 5463 & 3414 & 7125 \\
\hline 90 & 5089 & 5038 & 5517 & 5967 & 4946 \\
\hline 100 & 5705 & 5711 & 5379 & 5538 & 7047
\end{tabular}

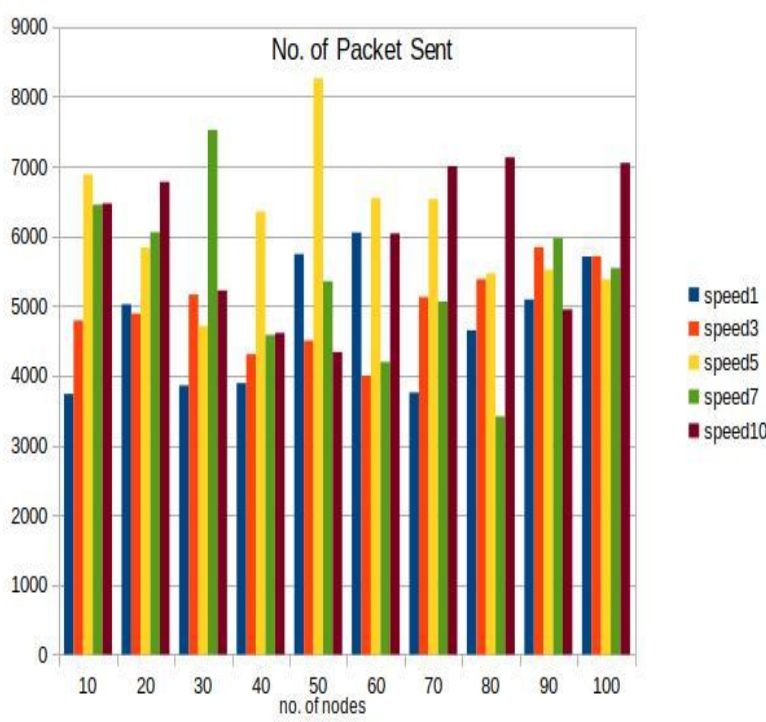

Fig 9: Simulation graph for No. of Packet sent

\subsection{For Packet Delivery Ratio}

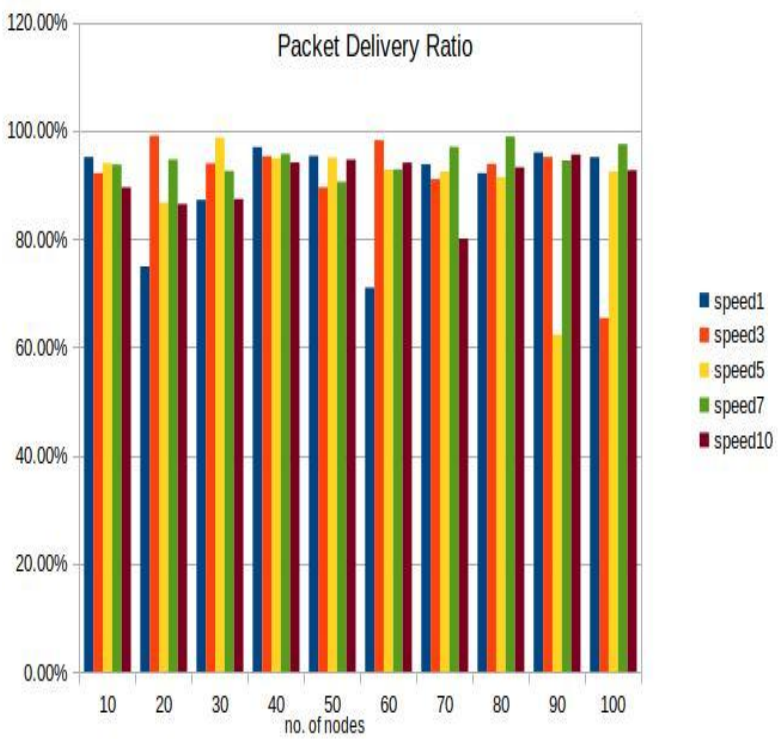

Fig 10: Simulation graph for Packet Delivery Ratio

Table 5. Simulation Result for No of packet delivery ratio

\begin{tabular}{|r|r|r|r|r|r|}
\hline 10. of nodes speed1 & \multicolumn{2}{|c|}{ speed3 } & \multicolumn{2}{|c|}{ speed5 } & \multicolumn{2}{l|}{ speed7 } & \multicolumn{2}{l|}{ speed10 } \\
\hline 10 & $95.13 \%$ & $92.17 \%$ & $94.01 \%$ & $93.73 \%$ & $89.50 \%$ \\
\hline 20 & $74.90 \%$ & $99.10 \%$ & $86.71 \%$ & $94.68 \%$ & $86.45 \%$ \\
\hline 30 & $87.19 \%$ & $93.97 \%$ & $98.70 \%$ & $92.54 \%$ & $87.35 \%$ \\
\hline 40 & $96.97 \%$ & $95.28 \%$ & $94.93 \%$ & $95.74 \%$ & $94.12 \%$ \\
\hline 50 & $95.33 \%$ & $89.53 \%$ & $95.04 \%$ & $90.54 \%$ & $94.65 \%$ \\
\hline 60 & $70.98 \%$ & $98.25 \%$ & $92.77 \%$ & $92.85 \%$ & $94.10 \%$ \\
\hline 70 & $93.77 \%$ & $91.02 \%$ & $92.46 \%$ & $97.02 \%$ & $80.03 \%$ \\
\hline 80 & $92.17 \%$ & $93.55 \%$ & $91.41 \%$ & $98.92 \%$ & $93.24 \%$ \\
\hline 90 & $95.97 \%$ & $95.12 \%$ & $62.28 \%$ & $94.49 \%$ & $95.59 \%$ \\
\hline 100 & $95.11 \%$ & $65.40 \%$ & $92.47 \%$ & $97.51 \%$ & $92.68 \%$ \\
\hline
\end{tabular}




\subsection{For Control Overhead}

Table 6. Simulation Result for Control overhead

\begin{tabular}{|c|c|c|c|c|c|}
\hline mo of nodes speedl & speed/3 & speed 5 & speed? & & v110 \\
\hline 10 & 190 & 173 & 193 & 200 & 102 \\
\hline 20 & 496 & 181 & 272 & 211 & 315 \\
\hline 30 & 737 & 353 & 249 & 305 & 314 \\
\hline 40 & 667 & 596 & 340 & 300 & 454 \\
\hline 50 & 783 & 484 & 34 & 550 & 24 \\
\hline 60 & 1833 & 401 & 848 & 52 & 47 \\
\hline 70 & 945 & 979 & 912 & 485 & 1069 \\
\hline 80 & 1821 & 701 & 654 & 703 & 581 \\
\hline 90 & 1770 & 560 & 1594 & 563 & 600 \\
\hline 100 & 1196 & 1434 & 928 & 641 & 894 \\
\hline
\end{tabular}

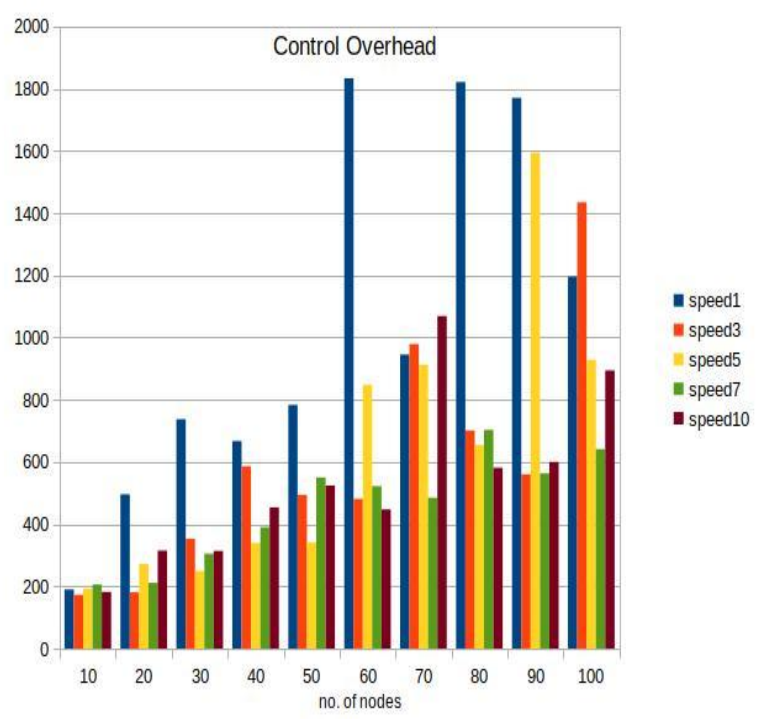

Fig 11: Simulation graph for Control Overhead

\subsection{For Normalized routing overhead}

Table 7. Simulation Result for Normalized routing overhead

\begin{tabular}{|r|r|r|r|r|r|}
\hline no. of nodes & \multicolumn{1}{l}{ speed1 } & \multicolumn{1}{l|}{ speed3 } & \multicolumn{1}{l|}{ speed5 } & \multicolumn{1}{l|}{ speed7 } & \multicolumn{1}{l|}{ speed10 } \\
\hline 10 & $5.35 \%$ & $3.92 \%$ & $2.98 \%$ & $3.41 \%$ & $3.14 \%$ \\
\hline 20 & $13.19 \%$ & $3.74 \%$ & $5.38 \%$ & $3.68 \%$ & $5.38 \%$ \\
\hline 30 & $21.91 \%$ & $7.28 \%$ & $5.35 \%$ & $4.38 \%$ & $6.89 \%$ \\
\hline 40 & $17.69 \%$ & $14.29 \%$ & $5.64 \%$ & $8.89 \%$ & $10.47 \%$ \\
\hline 50 & $14.31 \%$ & $12.26 \%$ & $4.34 \%$ & $11.35 \%$ & $12.77 \%$ \\
\hline 60 & $42.70 \%$ & $12.25 \%$ & $13.98 \%$ & $13.41 \%$ & $7.87 \%$ \\
\hline 70 & $26.84 \%$ & $20.99 \%$ & $15.11 \%$ & $9.88 \%$ & $19.08 \%$ \\
\hline 80 & $42.52 \%$ & $13.89 \%$ & $13.10 \%$ & $20.82 \%$ & $8.75 \%$ \\
\hline 90 & $36.24 \%$ & $10.08 \%$ & $46.39 \%$ & $9.99 \%$ & $12.69 \%$ \\
\hline 100 & $22.04 \%$ & $38.39 \%$ & $18.66 \%$ & $11.87 \%$ & $13.69 \%$ \\
\hline
\end{tabular}

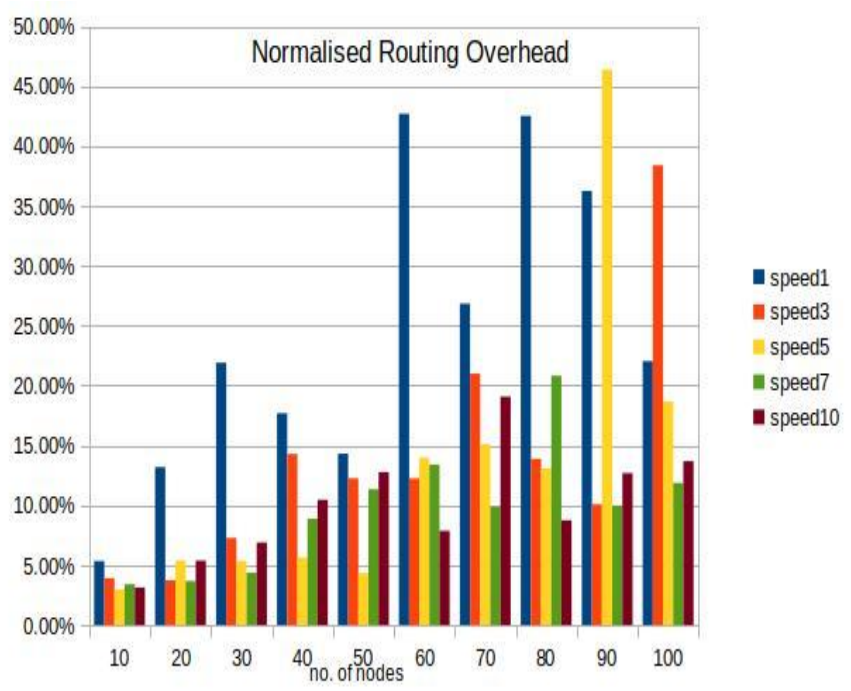

Fig 12: Simulation graph for Normalised Routing Overhead

\subsection{For Delay}

Table 8. Simulation Result for Delay

\section{no. of nodes speed1 speed3 speed5 speed7 speed10}

\begin{tabular}{|c|c|c|c|c|c|}
\hline 10 & 0.02 & 0.01 & 0.01 & 0.01 & 0.01 \\
\hline 20 & 0.02 & 0.01 & 0.01 & 0.01 & 0.01 \\
\hline 30 & 0.04 & 0.01 & 0.01 & 0.01 & 0.01 \\
\hline 40 & 0.02 & 0.02 & 0.01 & 0.01 & 0.08 \\
\hline 50 & 0.01 & 0.02 & 0.01 & 0.02 & 0.02 \\
\hline 60 & 0.01 & 0.02 & 0.09 & 0.01 & 0.01 \\
\hline 70 & 0.09 & 0.07 & 0.05 & 0.01 & 0.06 \\
\hline 80 & 0.22 & 0.01 & 0.01 & 0.03 & 0.01 \\
\hline 90 & 0.18 & 0.01 & 0.1 & 0.01 & 0.01 \\
\hline 100 & 0.01 & 0.02 & 0.02 & 0.01 & 0.01 \\
\hline
\end{tabular}

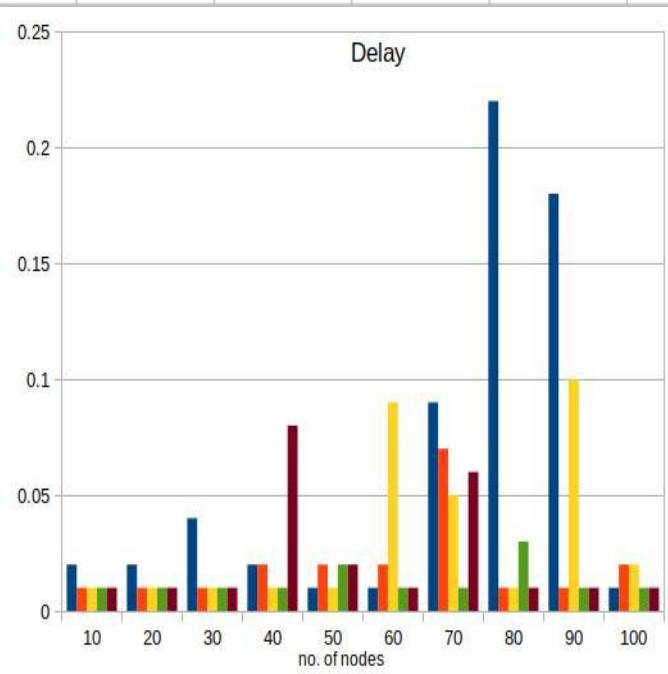

- speed1

inspeed 3

IIspeed5

speed7

- speed10

Fig 13: Simulation graph for Delay 
6.8 For Packet Dropping Ratio

Table 9. Simulation Result For Packet Dropping Ratio

\begin{tabular}{|r|r|r|r|r|r|}
\hline no. of nodes speed1 & \multicolumn{2}{|c|}{ speed3 } & \multicolumn{2}{c|}{ speed5 } & \multicolumn{2}{c|}{ speed7 } & \multicolumn{2}{l}{ speed10 } \\
\hline 10 & $4.87 \%$ & $7.83 \%$ & $5.99 \%$ & $6.27 \%$ & $10.50 \%$ \\
\hline 20 & $25.10 \%$ & $0.90 \%$ & $13.29 \%$ & $5.32 \%$ & $13.55 \%$ \\
\hline 30 & $12.81 \%$ & $6.03 \%$ & $1.23 \%$ & $7.46 \%$ & $12.65 \%$ \\
\hline 40 & $3.03 \%$ & $4.72 \%$ & $5.07 \%$ & $4.26 \%$ & $5.88 \%$ \\
\hline 50 & $4.67 \%$ & $10.47 \%$ & $4.96 \%$ & $9.46 \%$ & $5.35 \%$ \\
\hline 60 & $29.02 \%$ & $1.75 \%$ & $7.23 \%$ & $7.15 \%$ & $5.90 \%$ \\
\hline 70 & $6.23 \%$ & $8.98 \%$ & $7.54 \%$ & $2.98 \%$ & $19.97 \%$ \\
\hline 80 & $7.83 \%$ & $6.15 \%$ & $8.59 \%$ & $1.08 \%$ & $6.76 \%$ \\
\hline 90 & $4.03 \%$ & $0.88 \%$ & $37.72 \%$ & $5.51 \%$ & $4.41 \%$ \\
\hline 100 & $4.89 \%$ & $34.60 \%$ & $7.53 \%$ & $2.499 \%$ & $7.32 \%$ \\
\hline
\end{tabular}

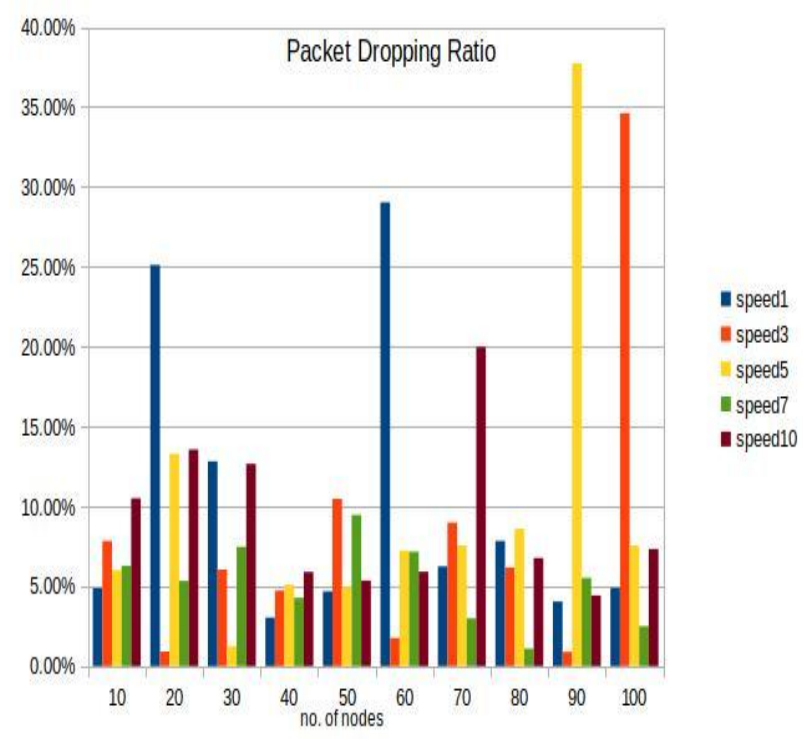

Fig 14: Simulation graph for Packet Dropping Ratio

\section{ANALYSIS}

In observation of simulation with NS-2, we found non linear change in the values of the matrices like Throughput, Number of packets sent, Number of packets received, Packets delivery ratio, Control overhead, Packet dropping ratio, Delay, Normalized routing overhead.

\section{CONCLUSION}

Thus we have evaluated the performance of very popular on demand routing protocol AODV, by means of various performance metrics such as Throughput, Number of packets sent, Number of packets received, Packet delivery ratio, Control overhead, Packet dropping ratio, Delay, Normalised routing overhead as well obtained simulation results by varying number of nodes in the network $\&$ speed $\&$ found that there is non linear change in the values of these metrics.

\section{REFERENCES}

[1] Bansal, R and Goyal, H, Jan 2011. Analytical Study the performance Evaluation of Mobile Adhoc Network using AODV Protocol. International Journal of Computer Application.

[2] Subramanya Bhat, M and Shwetha, D. August 2011. A Performance Study of Proactive, Reactive and Hybrid Routing Protocols using Qualnet Simulator, International Journal of Computer Applications ,0975 - 8887,v. 28 no.5.

[3] Agarwal, S, Krishnamurthy, S.V, Katz, R.H. and Dao, S. K, 2001. Distributed power control in ad-hoc wireless networks, Proc. of IEEE International Symposium on Personal, Indoor, and Mobile Radio Communications, San Diego.

[4] Chen, K, Shan, S. H. and Nahrstedt, K. April 2002, Cross-Layer Design for Data Accessibility in Mobile Adhoc Networks, Wireless Personal Communications, vol. 21, no. 1, pp. 49-76.

[5] Gandhewar, N and Patel. R. 2011, Performance Evaluation of AODV protocol in MANET using NS2 Simulator, 2nd National Conference on Information and Communication Technology (NCICT), Proceedings published in International Journal of Computer Applications.

[6] Ramachandran, B. and Shanmugan, S. 2006, Reliable Route Discovery for Mobile Ad-hoc Networks: A Crosslayer Approach, Proc. of IETE International Conference on Next Generation Networks, pp. 26.1-26.6.

[7] Bhati, P, Chauhan, R and Rathy, R. K, July 2011. An efficient Agent based AODV Routing Protocol in MANET, International Journal on Computer Science \& Engineering, v.7.

[8] Gupta, S.K and Saket, R. K. June 2011, Performance metric comparison of AODV and DSDV routing protocols in manets using ns-2, IJRRAS, v.7 (3).

[9] IBRAHIM, M and NEGLIA,G. Introduction to Network Simulator.

[10] Klein-Berndt, L. A Quick Guide to AODV Routing, Wireless Communication technology group National Institute of standard \& Technology.

[11] Muqattash, A and Krunz, M. 2003, Power Controlled Dual Channel (PCDC) Medium Access Protocol for Wireless Ad-hoc Networks, Proc. of IEEE INFOCOM.

[12] Feeney, L.M and Nilsson, M. April 2001, Investigating the Energy Consumption of a Wireless Network Interface in an Ad-hoc Networking Environment, Proc. of IEEE INFOCOM, 1548- 1557.

[13] Chandran, K, Raghunathan, S, Venkatesan, S and Prakash, R. February 2001, A Feedback-based Scheme for Improving TCP Performance in Ad-hoc Wireless Networks, IEEE Trans. on Personal Communications, 34-39, 